\title{
Prevalencia de asimetrías mandibulares en pacientes de Quito Ecuador medidas mediante radiografías panorámicas*
}

Prevalence of Mandibular Asymmetries in Patients from Quito Ecuador Measured through

Panoramic Radiographies

Fecha de recepción: 16-04-2018 | Fecha de aceptación: 11-11-2018

KAREM MARIANETH BARRENO HARO ${ }^{\mathrm{a}}$

Ministerio de Salud Pública, Puyo, Ecuador. karembarreno95@gmail.com.

https://orcid.org/0000-0002-1151-5056

Sandra Magdalena Macías Ceballos

Universidad Central del Ecuador, Quito, Ecuador.jimmacec@yahoo.com. https://orcid.org/0000-

$\underline{0002-2175-7710}$

*Investigación original.

a'Correspondencia: karembarreno95@gmail.com

doi: $\underline{\text { https://doi.org/10.11144/Javeriana.uo37-79.pamp }}$ 
Cómo citar: Barreno Haro KM, Macías Ceballos SM. Prevalencia de asimetrías mandibulares en pacientes de Quito Ecuador medidas mediante radiografías panorámicas. Univ Odontol. 2018 jul-dic; 37(79). https://doi.org/10.11144/Javeriana.uo37-79.pamp

\section{RESUMEN}

Antecedentes: Las asimetrías mandibulares se consideran variaciones morfológicas que pueden ser imperceptibles o asociarse a maloclusiones, trastornos temporomandibulares y asimetrías faciales. Objetivo: Determinar la prevalencia de asimetría vertical mandibular en radiografías panorámicas de pacientes atendidos en el centro quirúrgico de la Facultad de Odontología de la Universidad Central del Ecuador, mediante el análisis de Habets. Métodos: Se llevó a cabo un estudio descriptivo retrospectivo para analizar radiografías panorámicas de pacientes mayores de 15 años que presentaron dentición definitiva completa y asistieron a consulta durante los 3 últimos años. La muestra estuvo compuesta por 680 radiografías que correspondían 309 a pacientes hombres y 371 mujeres, con una edad promedio de 22,02 años. Se utilizaron la prueba de Chi cuadrado para evaluar la asociación de las asimetrías encontradas en cada una de las variables dependientes con la edad y sexo del paciente, y las de Mann-Whitney y Kruskal Wallis para la distribución de las variables (intervalo de confianza del $95 \%$, p $\leq 0,05$ ). Resultados: La prevalencia de asimetrías verticales condilares fue del 70,3\%, las de rama 38,7 \% y la de cóndilo-rama 30,7 \%. Estos valores presentaron mayor tendencia hacia el lado izquierdo. Conclusión: El índice de asimetría de Habets con un corte del $3 \%$ mostró una prevalencia de asimetría condilar en mayor grado, seguida por la asimetría de rama y, menos prevalente, la cóndilo-rama. Estos valores no representaron significancia estadística en relación con la edad y el sexo del paciente. 


\section{Palabras clave}

análisis de Habets; asimetría condilar; asimetría de cóndilo-rama; asimetría de rama; asimetría vertical mandibular; cóndilo; Ecuador; Quito; radiografía panorámica; rama

\section{Áreas temáticas}

cirugía oral y maxilofacial; odontología; ortodoncia; rehabilitación oral

\section{ABSTRACT}

Background: Mandibular asymmetries are considered morphological variations, which may be imperceptible or linked to malocclusions, temporomandibular disorders, and facial asymmetries. Purpose: To determine the prevalence of mandibular vertical asymmetries through panoramic radiographs of patients treated at the surgical center of the Universidad Central del Ecuador using Habets analysis. Methods: A descriptive and retrospective was conducted to analyze panoramic radiographies of older-than-15-year-old patients who presented complete permanent dentition and were seen within the last 3 years. The sample consisted of 680 radiographs of 309 men and 371 women, with an average age of 22.02 years. Chi-square test was used to analyze the association between asymmetries found for each dependent variable and patients' age and sex. Mann-Whitney and Kruskal Wallis tests were performed to analyze variable distribution (confidence interval of $95 \%$. p $\leq 0.05)$. Results: The prevalence of vertical condylar asymmetry was $70.3 \%$, followed by ramus $(38.7 \%)$, and condyle- ramus $(30.7 \%)$. There was a larger tendency towards the left side. Conclusion: The Habets asymmetry index with a $3 \%$ cut showed a prevalence of condylar 
asymmetry to a greater degree, followed by ramus asymmetry and condylar-ramus asymmetry. These values did not show statistical significance regarding age and sex.

\section{Keywords}

Condylar assymetry; condyle; condyle-ramus assymetry; Ecuador; Habets analysis; panoramic radiograph; Quito; vertical mandibular asymmetry; ramus; ramus assymetry

\section{Thematic fields}

dentistry; oral and maxillofacial surgery; oral rehabilitation; orthodontics

\section{INTRODUCCIÓN}

Las asimetrías mandibulares han sido consideradas variaciones morfológicas que indican desproporción en su forma, tamaño o posición entre el lado derecho e izquierdo (Okeson, 2013; Sheikhi, Ghazizadeh, \& Mahdi, 2017; Navarro, 2009), estas pueden ser imperceptibles sin llegar a influir en función y estética o por el contrario vincularse con alteraciones como maloclusiones, trastornos temporomandibulares (TTM), asimetrías faciales y hasta afectar el desarrollo psicosocial del paciente causando inconvenientes para incluirse en la sociedad (Navarro, 2009; Halicioğlu, Celikoglu, Buyuk, Sekerci, \& Candirli, 2014; Kasimoglu, Tuna, Rahimi, Marsan, \& Gencay, 2014; Alfar, Ayala, Barrientos, \& Rodríguez, 2016). Por lo que es esencial su identificación de manera temprana y lo podemos hacer mediante una herramienta rutinariamente utilizada por los Odontólogos como la radiografía panorámica (Habets L, 1987; Habets, Bezuur, Naeiji, \& Hansson, 1988). 
La mandíbula consta de dos hemiarcadas semejantes, cada una de ellas tiene una articulación independiente y se encuentran unidas por la sínfisis, es por ello que pueden crecer de forma aislada una de la otra, ya sea que un lado presente déficit o demasía de crecimiento nos dará como resultado una asimetría mandibular. Navarro (Navarro, 2009) indica en su texto publicado en el 2009 que la porción condilar de la mandíbula ha sido la responsable del crecimiento mandibular, coincidiendo con Sezguin y colaboradores (Sezgin, Celenkb, \& Aricic, 2007) por lo que han considerado al cóndilo como un regulador del crecimiento, atribuyéndole ser una de las causas principales de la asimetría mandibulofacial.

Estudios recientes de Kasimoglu y colaboradores (Kasimoglu, Tuna, Rahimi, Marsan, \& Gencay, 2014) en el 2014 han vinculado la presencia de maloclusiones al desarrollo de una asimetría condilar, indicando que el desbalance oclusal puede conllevar a un cambio morfológico en el cóndilo y por ende podría producir una asimetría mandibular; dentro de las maloclusiones podemos destacar la mordida cruzada posterior durante el crecimiento reportada por Arenas (Arenas, Araya, \& Palomino, 2012) en el 2012 que ha sido considerada como un importante factor de riesgo.

Por otra parte, la asimetría condilar también ha sido considerada como un posible factor causal para el desarrollo de TTM. Habets y colaboradores (Habets L, 1987; Habets, Bezuur, Naeiji, \& Hansson, 1988), tras la creación del índice de asimetría mandibular vertical, en 1988 afirmaron que es un factor estructural que debería ser evaluado en el diagnóstico, ya que asociado a otros signos y síntomas aumenta la posibilidad de adquirir un TTM (Okeson, 2013; Habets L, 1987; Fuentes, Silva, Sandoval, Cuevas, \& Rodríguez, 2006). Mostrando concordancia con lo encontrado 
por Bezuur (Bezuur, Habets, \& Hansson, 1988), quién concluyó que el 74 \% de pacientes con TTM presentaron asimetría condilar.

Es por ello que cabe destacar la importancia de su correcto diagnóstico, que puede ser realizado de forma inicial mediante radiografías panorámicas, ya que es una técnica en donde el paciente no se expone a altos índices de radiación en comparación con otras y además es asequible para los pacientes por su costo conveniente; por otro lado todos los profesionales odontólogos pueden manejar este tipo de radiografía por lo que es comúnmente utilizada en la consulta, gracias a que brinda una visión general de la salud bucal del paciente (Kasimoglu, Tuna, Rahimi, Marsan, \& Gencay, 2014).

Además en la presente investigación se aplicó el índice de Habets que nos indica la presencia o no de asimetría vertical mandibular; este análisis compara la altura del cóndilo, la rama y cóndilo más rama mandibular derecho e izquierdo por medio de la proyección de puntos y planos anatómicos que pueden ser medidos usando el trazado radiográfico manual de forma rápida y sencilla, cabe señalar que Habets realizó estudios de las tomas radiográficas mediante un estudio experimental con anterioridad para elaborar una fórmula precisa en donde la distorsión de la radiografía panorámica ha sido controlada (Habets L, 1987; Habets, Bezuur, Naeiji, \& Hansson, 1988).

En Ecuador no se han encontrado aportes investigativos sobre este tema a pesar de su diagnóstico asequible mediante dicho análisis y su importancia, teniendo en cuenta que el principal motivo de consulta en ortodoncia es estético y omitir una alteración esquelética podría llevar a un tratamiento sin resultados satisfactorios tanto para el paciente como para el profesional, sin contar los riesgos 
que se pueden producir en el paciente el no identificar este tipo de alteraciones (Sheikhi, Ghazizadeh, \& Mahdi, 2017; Galarraga, 2000).

Por esto se realizó el presente estudio, con la finalidad de conocer la prevalencia de asimetría vertical mandibular en las radiografías panorámicas de los pacientes mayores de 15 años que presentaron dentición definitiva completa, atendidos en el Centro Quirúrgico de la Facultad de Odontología de la Universidad Central del Ecuador (UCE) durante el período enero 2015diciembre 2017.

\section{MATERIALES Y MÉTODOS}

Se usó una metodología observacional, retrospectiva y descriptiva, para analizar radiografías panorámicas de pacientes mayores de 15 años que presentaron dentición definitiva completa y fueron atendidos durante el período enero 2015 - diciembre 2017 en el centro quirúrgico de la Facultad de Odontología de la UCE. La muestra del presente estudio estuvo conformada por 680 radiografías panorámicas que reposan en las historias clínicas de dicho centro y cumplieron con los criterios de inclusión; las cuales cuentan con sus respectivos consentimientos informados firmados previamente por los pacientes en donde aceptaron que sus radiografías pueden ser utilizadas con fines investigativos de manera confidencial.

Tras su análisis se incluyeron únicamente las radiografías panorámicas de pacientes atendidos en el Centro Quirúrgico de la Facultad de Odontología de la UCE mayores de 15 años de edad, que presentaron dentición definitiva completa con o sin terceros molares, sin tratamiento de ortodoncia 
y con radiografías de excelente calidad diagnóstica. Asimismo se excluyeron las radiografías panorámicas en donde los cóndilos o rama ascendente de la mandíbula no fueron enfocados, además de las imágenes que presentaron daños físicos como manchas o roturas (figura 1). Con todos estos requisitos y tras la aprobación del Comité de ética de la UCE se procedió a evaluar las radiografías panorámicas con el índice de Habets (Habets, Bezuur, Naeiji, \& Hansson, 1988) mediante trazado radiográfico manual, considerando los siguientes valores:

- CH: altura condilar vertical, que corresponde a la distancia medida en milímetros desde el punto más superior del cóndilo, representado por el punto de intersección formado por la línea A y B, hasta el punto más lateral del cóndilo de la mandíbula $\left(\mathrm{O}_{1}\right)($ Habets, Bezuur, Naeiji, \& Hansson, 1988).

- RH: altura vertical de la rama mandibular que corresponde a la distancia medida en milímetros desde el punto más lateral del cóndilo de la mandíbula $\left(\mathrm{O}_{1}\right)$ hasta el punto más lateral de la rama ascendente de la mandíbula $\left(\mathrm{O}_{2}\right)$ (Habets, Bezuur, Naeiji, \& Hansson, 1988).

- Altura de cóndilo más rama: indica la distancia vertical que muestra un dato general de asimetría mandibular, se mide desde el punto de intersección formado por línea A y B, hasta el punto más lateral de la rama ascendente de la mandíbula $\left(\mathrm{O}_{2}\right)$; o suma de los dos valores anteriores (Habets, Bezuur, Naeiji, \& Hansson, 1988). 


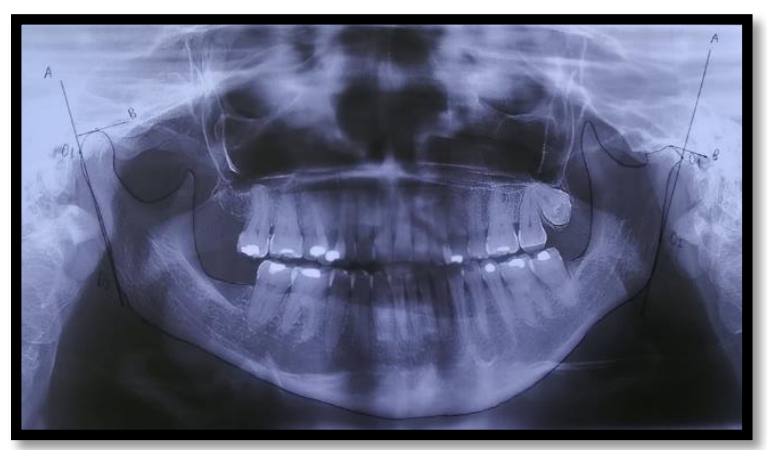

Una vez trazados los puntos y planos propuestos por Habets, con la ayuda de un negatoscopio de luz LED, se midieron las distancias descritas y con los valores obtenidos se aplicó la fórmula de asimetrías [ ( R - L ) / ( R + L ) ] x $100 \%$; donde R corresponde al lado derecho de la estructura medida y L al lado izquierdo. Esta operación nos llevó a diagnosticar la simetría o asimetría de los cóndilos, rama y cóndilo más rama mediante valores porcentuales, en donde un valor menor o igual a $3 \%$ nos indica una simetría y mayor a este una asimetría de las estructuras en estudio (Habets, Bezuur, Naeiji, \& Hansson, 1988).

Cabe señalar que este valor de corte fue incorporado por Habets y colaboradores (Habets L, 1987) en el año 1987, para identificar asimetrías mandibulares en radiografías panorámicas. Tras varios análisis que se enfocaron en reducir la distorsión producida por la radiografía panorámica en la toma radiográfica, para lo cual usaron un modelo experimental que se basó en un bloque que simulaba a una mandíbula y de forma especial a cóndilos, los mismos que fueron dispuestos a diferentes alturas. 
Este bloque fue sometido a varias tomas radiográficas en 9 diferentes posiciones, simulando de esta manera el posible cambio de posición del paciente durante la toma radiográfica, obteniendo como resultado, que las asimetrías de los cóndilos simuladas que presentaron una diferencia del 6 \% pueden ser aceptables con respecto a variaciones de $10 \mathrm{~mm}$ en la posición de la cabeza (Habets L, 1987; Habets, Bezuur, Naeiji, \& Hansson, 1988).

Lo que dio paso a la elaboración del índice de asimetrías en donde se estableció que el $6 \%$ de diferencia entre los cóndilos del modelo experimental resulta un 3\% de asimetría en la fórmula antes descrita, estableciendo un valor mayor a 3 como el punto de corte para establecer o no una asimetría vertical en el cóndilo, rama o cóndilo más rama (Habets, Bezuur, Naeiji, \& Hansson, 1988). Es por ello que Van Elslande y colaboradores (Van Elslande, Russett, Major, \& Flores-Mir, 2008) en su publicación, consideran a este método válido para determinar asimetrías mandibulares y es utilizado hasta la actualidad por diversos autores de artículos científicos que han sido publicados en prestigiosas revistas (Kasimoglu, Tuna, Rahimi, Marsan, \& Gencay, 2014; Arenas, Araya, \& Palomino, 2012; Fuentes, Silva, Sandoval, Cuevas, \& Rodríguez, 2006).

Antes de que la metodología sea aplicada por el operador, se llevó a cabo la estandarización, en donde dos examinadores especialistas en Ortodoncia, calibraron en una sesión teórica a la Investigadora en la identificación de los puntos y planos propuestos por Habets para el diagnóstico de asimetrías mandibulares (Habets, Bezuur, Naeiji, \& Hansson, 1988), con el fin de que exista una correcta aplicación del mismo por parte del operador. 
Posterior a ello se realizó una prueba práctica para evaluar la concordancia entre observadores bajo la asesoría de los mismos especialistas, en donde se analizaron 24 radiografías panorámicas facilitadas por un Centro Odontológico particular que fueron evaluadas con el índice propuesto, por cada operador de manera independiente y se obtuvo un resultado del coeficiente de concordancia intraclase promedio de 0,76 considerado como "bueno". Se aseguró de esta manera una correcta interpretación y un registro adecuado de las muestras por parte de la investigadora; cabe mencionar que estas radiografías no se incluyeron en el estudio ya que no formaron parte de la muestra.

Los resultados obtenidos en el estudio fueron recopilados en una hoja de cálculo de Microsoft Excel versión 2013 en donde se ubicaron los datos de las variables dependientes (cóndilo, rama y cóndilo más rama tanto derechos como izquierdos) e independientes (sexo y edad). Posterior a ello también se analizó mediante valores numéricos y porcentuales para cada variable, empleando el programa estadístico SPSS versión 22. Se usó un intervalo de confianza del $95 \%$ ( $\mathrm{p}<0.5)$ para las siguientes pruebas estadísticas: chi cuadrado de Pearson para la realización de tablas cruzadas entre cada una de las variables con la de edad y sexo respectivamente, y pruebas no paramétricas de Mann Whitney y Kruskal Wallis para la distribución de variables comparada con sexo y grupos etarios respectivamente.

\section{RESULTADOS}

De las 680 radiografías valoradas se encontró una edad media de 22,02 años que correspondió el $54,6 \%(n=371)$ a mujeres y el 45,4\% (n=309) a hombres. Al obtener los grupos etarios se encontró 
que el más prevalente fue el correspondiente a las radiografías panorámicas de pacientes que tuvieron entre 20 a 24 años con un porcentaje de 48,5\% (n=330), seguidos de los pacientes que presentaron entre 15 a 19 años con el 30,3\% (n=206) y por último el grupo conformado por los pacientes que tuvieron 25 a más años con el 21,2\% $(\mathrm{n}=144)$.

Al aplicar el índice de asimetría de Habets con un corte del $3 \%$ existió prevalencia de asimetría condilar en el plano vertical del 70,3\% $(\mathrm{n}=478)$, seguida por la asimetría de rama del 38,7 \% (n=263) y por último la menos prevalente fue de cóndilo más rama del 30,7 \% (n = 209); estos valores presentaron mayor tendencia hacia el lado izquierdo y no presentaron significancia en relación a la edad y sexo del paciente (tablas 1 y 2).

TABLA 1

PREVALENCIA DE ASIMETRÍA MANDIBULAR

\begin{tabular}{lcccccc}
\hline & \multicolumn{2}{c}{ Asimetría Condilar } & \multicolumn{2}{c}{ Asimetría Rama } & \multicolumn{2}{c}{ Asimetría Cóndilo-Rama } \\
& Frecuencia & Porcentaje & Frecuencia & Porcentaje & Frecuencia & Porcentaje \\
\cline { 3 - 7 } Simetría & 202 & 29,7 & 417 & 61,3 & 471 & 69,3 \\
Asimetría & 478 & 70,3 & 263 & 38,7 & 209 & 30,7 \\
Total & 680 & 100,0 & 680 & 100,0 & 680 & 100,0 \\
\hline
\end{tabular}

TABLA 2

DISTRIBUCIÓN DE ASIMETRÍAS

\begin{tabular}{lcccccc}
\hline \multirow{2}{*}{ Asimetría } & \multicolumn{2}{c}{ Cóndilo } & \multicolumn{2}{c}{ Rama } & \multicolumn{2}{c}{ Cóndilo-Rama } \\
& Frecuencia & Porcentaje & Frecuencia & Porcentaje & Frecuencia & Porcentaje \\
\hline Derecho & 221 & 46,23 & 97 & 36,88 & 79 & 37,80 \\
Izquierdo & 257 & 53,77 & 166 & 63,12 & 130 & 62,20 \\
Total & 478 & 100,00 & 263 & 100,00 & 209 & 100,00 \\
\hline
\end{tabular}

Además se evaluó únicamente las medidas obtenidas en milímetros de las variables dependientes, en donde se encontró que la medida promedio en milímetros en los cóndilos de las pacientes de sexo femenino fue de $6,6 \mathrm{~mm}$ y masculino de $6,5 \mathrm{~mm}$; seguidas de las medidas promedio en milímetros encontradas en la rama que fueron de 42,5 mm en mujeres y 47,4 mm en hombres; y 
por último las medidas promedio en milímetros encontradas en la distancia cóndilo más rama que fue de 49,2 mm para mujeres y 53,9 mm para hombres, lo que ha indicado que la altura vertical de la rama y cóndilo más rama fue mayor en hombres que en mujeres (tabla 3).

TABLA 3

COMPARACIÓN DE ALTURA EN MILÍMETROS POR SEXO

\begin{tabular}{|c|c|c|c|c|c|c|c|}
\hline & & $\mathbf{N}$ & Media & $\begin{array}{l}\text { Desviación } \\
\text { estándar }\end{array}$ & $\begin{array}{c}\text { Error } \\
\text { estándar }\end{array}$ & Mínimo & Máximo \\
\hline \multirow[t]{3}{*}{$\overline{C D}$} & \multirow{3}{*}{$\begin{array}{l}\text { Masculino } \\
\text { Femenino } \\
\text { Total }\end{array}$} & 309 & 6,50 & 2,282 & 0,130 & 2 & 13 \\
\hline & & 371 & 6,60 & 2,206 & 0,115 & 2 & 16 \\
\hline & & 680 & 6,55 & 2,240 & 0,086 & 2 & 16 \\
\hline \multirow[t]{3}{*}{ CI } & \multirow{3}{*}{$\begin{array}{l}\text { Masculino } \\
\text { Femenino } \\
\text { Total }\end{array}$} & 309 & 6,57 & 2,352 & 0,134 & 2 & 13 \\
\hline & & 371 & 6,74 & 2,292 & 0,119 & 2 & 16 \\
\hline & & 680 & 6,66 & 2,319 & 0,089 & 2 & 16 \\
\hline \multirow[t]{3}{*}{ RD } & Masculino & 309 & 47,06 & 6,212 & 0,353 & 32 & 61 \\
\hline & Femenino & 371 & 42,34 & 5,428 & 0,282 & 27 & 58 \\
\hline & Total & 680 & 44,49 & 6,251 & 0,240 & 27 & 61 \\
\hline \multirow[t]{3}{*}{ RI } & Masculino & 309 & 47,65 & 6,459 & 0,367 & 29 & 70 \\
\hline & Femenino & 371 & 42,73 & 5,754 & 0,299 & 26 & 60 \\
\hline & Total & 680 & 44,96 & 6,557 & 0,251 & 26 & 70 \\
\hline \multirow[t]{3}{*}{ C+RD } & Masculino & 309 & 53,55 & 6,882 & 0,392 & 37 & 73 \\
\hline & Femenino & 371 & 48,94 & 6,337 & 0,329 & 31 & 67 \\
\hline & Total & 680 & 51,04 & 6,976 & 0,268 & 31 & 73 \\
\hline \multirow[t]{3}{*}{$\mathrm{C}+\mathrm{RI}$} & Masculino & 309 & 54,23 & 6,985 & 0,397 & 36 & 74 \\
\hline & Femenino & 371 & 49,47 & 6,476 & 0,336 & 29 & 68 \\
\hline & Total & 680 & 51,63 & 7,114 & 0,273 & 29 & 74 \\
\hline
\end{tabular}

$\mathrm{CD}=$ Cóndilo Derecho, $\mathrm{CI}=$ Cóndilo Izquierdo, $\mathrm{RD}=$ Rama Derecha, $\mathrm{RI}=$ Rama Izquierda, $\mathrm{C}+\mathrm{RD}=\mathrm{Cóndilo} \mathrm{más} \mathrm{rama}$ derechos, $\mathrm{C}+\mathrm{RI}=$ Cóndilo más rama izquierdos

\section{DISCUSIÓN}

En el presente estudio se obtuvo una alta prevalencia de asimetría condilar del 70,3\%, coincidiendo con valores obtenidos por los creadores del índice de asimetría en Ámsterdam, tales como Habets (Habets, Bezuur, Naeiji, \& Hansson, 1988) quien encontró asimetría en 109 de 152 pacientes, que corresponde al 71,71\% de su muestra y de manera similar Bezuur (Bezuur, Habets, \& Hansson, 1988), quien obtuvo un resultado significativo de $74 \%$, ambos resultados 
correspondieron a una muestra de pacientes que presentaron dentición definitiva al igual que nuestra muestra.

Además el resultado de asimetría condilar también mostró una coincidencia relativa con investigaciones realizadas en Chile por autores como Fuentes (Fuentes, Silva, Sandoval, Cuevas, \& Rodríguez, 2006) donde se obtuvo un 67,1 \% de prevalencia en una muestra formada por adolescentes con dentición mixta, al igual que Arenas (Arenas, Araya, \& Palomino, 2012) quien alcanzó una asimetría en 71 pacientes de 120 obteniendo un 58,67 \% de asimetría. Cabe destacar que no todos los pacientes seleccionados para estos estudios presentaron dentición definitiva, lo que podría relacionarse con los resultados que fueron ligeramente más bajos, sin embargo se habla de una población de Sudamérica la cual se acercaría más a nuestros rasgos étnicos.

Por el contrario no se obtuvo coincidencia con la investigación realizada en Turquía por Kasimoglu (Kasimoglu, Tuna, Rahimi, Marsan, \& Gencay, 2014), ya que obtuvo como resultado valores de asimetría condilar que oscilaron entre el 3,50\% y 9,49\%, estos valores diferentes pueden estar asociados a que su muestra fueron adolescentes con una edad media de 13,64 años, además que la población en estudio contó con rasgos distintos y cultura diferente a la nuestra.

En cuanto a la rama mandibular se obtuvo una prevalencia de 38,7 \%, valor que es muy próximo a lo obtenido por Bezuur (Bezuur, Habets, \& Hansson, 1988) con un $39 \%$ de asimetría; además nuestro resultado se encontró como un valor intermedio, comparado con lo publicado por Habets (Habets, Bezuur, Naeiji, \& Hansson, 1988) con una muestra de 31 pacientes de 151, correspondiente al 20,39\%, y con lo publicado por Arenas (Arenas, Araya, \& Palomino, 2012) en 
Chile, donde encontró una asimetría de rama en 64 pacientes de 121, que corresponde al 52,89 \% de su muestra.

La asimetría menos prevalente fue la de cóndilo más rama, con un 30,7 \%, valor similar al propuesto por Bezuur (Bezuur, Habets, \& Hansson, 1988) en el $26 \%$ del total de su muestra, seguido de la investigación realizada por Arenas en Chile (Arenas, Araya, \& Palomino, 2012) que encontró una asimetría en 27 pacientes de un total de 121, correspondiente al 22,31\% de su muestra y la investigación con la que menos hubo coincidencia fue la de Habets (Habets, Bezuur, Naeiji, \& Hansson, 1988) con 23 pacientes asimétricos de 152 que correspondieron al 15,13\% del total de su muestra.

También se evaluó la asociación de la asimetría encontrada en cada una de las variables dependientes antes mencionadas con la edad y sexo del paciente, en dónde no se encontró ninguna relación significativa, coincidiendo de esta forma con Habets y Alfaro (Alfar, Ayala, Barrientos, \& Rodríguez, 2016; Habets, Bezuur, Naeiji, \& Hansson, 1988) que tampoco encontraron influencia de la asimetría vertical mandibular con relación al género y edad, en contraste diferimos con Fuentes (Fuentes, Silva, Sandoval, Cuevas, \& Rodríguez, 2006) que encontró una asimetría significantemente mayor en el sexo femenino, se puede justificar este valor por la presencia de más mujeres en su muestra.

Se evaluaron únicamente las medidas lineales de las variables dependientes medidas en milímetros, sin aplicar la fórmula de Habets, en donde se estableció valores promedios del cóndilo, 
que presentó una altura de 6,6 $\mathrm{mm}$ en mujeres y de 5,5 $\mathrm{mm}$ en hombres, presentando una dimensión similar, a diferencia de las medidas obtenidas en la rama y cóndilo-rama.

En la rama se encontró una altura promedio de $42,5 \mathrm{~mm}$ en mujeres y $47,4 \mathrm{~mm}$ en hombres que indica una diferencia en relación al sexo, siendo la rama del hombre de mayor tamaño que las mujeres; de igual forma se pudo demostrar este resultado en la altura de cóndilo-rama acorde al sexo, con un promedio de 49,2 $\mathrm{mm}$ para mujeres y 53,9 $\mathrm{mm}$ para hombres, en donde también se presentó una altura vertical de cóndilo-rama mayor en hombres que en mujeres. En cuanto a la relación de las medidas de las variables con la edad del paciente no se demostró ninguna diferencia entre edades.

Los análisis de asimetrías mediante radiografías panorámicas ha sido un tema de controversia por la posible distorsión que pueda existir en sus imágenes, sin embargo varios estudios señalan que cambios en la posición de la cabeza del paciente pueden producir una distorsión que afecte a la dimensiones horizontal de la radiografía panorámica, mientras que el mismo suceso no produce grandes cambios en las dimensiones verticales $(5,12,14-16)$; mostrando de esta manera que se podrían realizar mediciones de asimetrías verticales, obteniendo medidas aceptables (Larheim \& Svanaes, 1986), tomando en cuenta además que al realizar la toma radiográfica cada equipo regula la posición del paciente, estandarizando el procedimiento.

Por otro lado, se sabe que el patrón para evaluar asimetrías ha sido la tomografía computarizada (Sanders, Rigali, Neace, Uribe, \& Nanda, 2010; Dawson, 2009) pero presenta desventajas como una alta dosis de radiación y costo elevado, por lo que su uso se ha recomendado para casos en los 
cuales exista alguna sospecha o quiera aclararse un diagnóstico, de igual manera sería recomendable que sean llevados por especialistas, ya que no todos los odontólogos generales tienen el conocimiento necesario para analizarla de una manera correcta.

Por ello el uso de la radiografía panorámica para identificar asimetrías verticales en la mandíbula es válido, ya que puede ser evaluada por cualquier odontólogo, presenta bajas dosis de radiación y tiene un costo accesible para el paciente; sabiendo además que ha sido reconocida como un examen complementario válido para diagnosticar una asimetría (Van Elslande, Russett, Major, \& Flores-Mir, 2008; Kambylafkas, Murdock, Gilda, Tallents, \& Kyrkanides, 2006; Türp, Vach, Harbich, Alt, \& Strub, 1996), teniendo en cuenta que no es un factor determinante, pero sí nos da una idea general del estado de salud oral del paciente y una posibilidad para dar un tratamiento temprano (Arenas, Araya, \& Palomino, 2012; Fuentes, Silva, Sandoval, Cuevas, \& Rodríguez, 2006).

Por la presencia de prevalencia de asimetría que se mostró en el estudio, especialmente a nivel condilar, se propone realizar este sencillo análisis que nos brinda un importante aporte diagnóstico para el paciente, y tener en cuenta que si se obtiene un resultado positivo, se debería realizar un examen clínico más detallado con otros elementos de apoyo y una valoración multidisciplinaria si fuera necesario, ya que una asimetría se considera un factor de riesgo para desarrollar un trastorno temporomandibular (Okeson, 2013; Kasimoglu, Tuna, Rahimi, Marsan, \& Gencay, 2014; Arenas, Araya, \& Palomino, 2012; Fuentes, Silva, Sandoval, Cuevas, \& Rodríguez, 2006; Dawson, 2009).

\section{CONCLUSIONES}


Tras analizar las radiografías panorámicas, en nuestro estudió se encontró una prevalencia significativa de asimetrías vertical de cóndilo del 70,3\%, seguido por una menor prevalencia en rama del 38,7 \% y cóndilo más rama con un 30,7 \% mostrando en cada estructura una ligera tendencia hacia el lado izquierdo. Así mismo se determinó que no hay diferencias estadísticas significativas en relación a las variables dependientes (Cóndilo, rama y cóndilo más rama derechos e izquierdos) con respecto a la edad y sexo del paciente.

\section{RECOMENDACIONES}

Se recomienda realizar más estudios sobre el tema y sus implicaciones diagnósticas en las diferentes áreas odontológicas, especialmente en Ortodoncia, Rehabilitación Oral y Cirugía Oral; para lo cual se puede incluir una valoración completa de la radiografía panorámica, integrando el índice de Habets para dar un diagnóstico integral al paciente, especialmente en la zona del cóndilo en donde existe mayor prevalencias de asimetrías reportadas en la literatura y que podría afectar la armonía de la ATM.

Además, se sugiere realizar investigaciones con otros índices de asimetría para verificar la validez de estos y determinar cuál de ellos es el más confiable, de modo que la radiografía panorámica sea explotada de la mejor manera como examen radiográfico de primera elección. De esta manera como profesionales odontólogos podríamos concientizar la idea de brindar al paciente un tratamiento integral, analizando cada componente del aparato estomatognático y lo que podría 
implicar una alteración de alguna de sus estructuras sea valorado de una manera más amplia y de ser necesario mediante otros exámenes complementarios.

\section{REFERENCIAS}

1. Okeson JP. Tratamiento de oclusión y afecciones temporomandibulares. $7 \mathrm{ma}$ ed. Madrid España: Elsevier; 2013.

2. Sheikhi M, Ghazizadeh M, Salehi MM. Posttraumatic mandibular asymmetry presenting in a young adult. Radiology Case Reports. 2017; I(2): 73-77. doi: $\underline{0.1016 / j . r a d c r .2016 .10 .023}$

3. Navarro C. Tratado de cirugía oral y maxilofacial. Tomo I. 2da ed. Madrid España: Arán; 2009.

4. Halicioğlu K, Celikoglu M, Buyuk SK, Sekerci AE, Candirli C. Effects of early unilateral mandibular first molar extraction on condilar and ramal vertical asymmetry. Eur J Dent. 2014 Apr-Jun; 8(2): 178-183.doi: 10.1007/s00784-012-0843-9

5. Kasimoglu Y, Tuna EB, Rahimi B, Marsan G, Gencay K. Condylar asymmetry in different occlusion types. Cranio. 2014 Jul; 0(0): 1-5. doi:10.1179/0886963414Z.00000000039

6. Alfaro C, Ayala R, Barrientos S, Rodríguez CA. Prevalencia de asimetrías mandibulares en radiografías panorámicas de población de Bogotá-Colombia. Int J Morphol. 2016 Ago; 34(4): 1203-1206. doi:dx.doi.org/10.4067/S0717-95022016000400004

7. Habets LL, Bezuur JN, VanOoij CP, Hansson TL. The orthopantomogram, an aid in diagnosis of temporomandibular joint problems. I. The factor of vertical magnification. J. Oral Rehabil. 1987; 14(5): 475-80. 
8. Habets LL, Bezuur JN, Naeiji M, Hansson TL. The orthopantomogram, an aid in diagnosis of temporomandibular joint problems. II. The vertical symmetry. J. Oral Rehabil. 1988; 15(5): 465-71.

9. Sezgin OS, Celenk P, Aricic S. Mandibular asymmetry in different occlusion patterns. Angle Orthod. 2007; 77(5): 803-7). doi: doi.org/10.2319/092506-392

10. Arenas CS, Araya P, Palomino MH. Evaluación de la asimetría vertical mandibular, en pacientes con mordida cruzada posterior uni y bilateral. Int J Morphol. 2012, Jun; 30(3):883-890. doi: dx.doi.org/10.4067/S0717-95022012000300020

11. Fuentes R, Silva H, Sandoval P, Cuevas F, Rodríguez M. Altura del proceso condilar en pacientes con diferentes clases esqueletales que requieren tratamiento de ortodoncia. Int $\mathbf{J}$ Morphol. 2006 Sep; 24(3): 499-503. doi:10.4067/S0717-95022006000400033

12. Bezuur JN, Habets LL, Hansson TL. The recognition of craniomandibular disorders - a comparison between clinical, tomographical, and dental panoramic radiographical findings in thirty-one subjects. J Oral Rehabil. 1988; 15(6): 549-554. doi:

\section{https://doi.org/10.1111/j.1365-2842.1988.tb00191.x}

13. Galarraga N. Motivo de consulta en el paciente ortodóncico adolescente. Acta odontol. venez. 2000; 38(1).

14. Van Elslande DC, Russett SJ, Major PW, Flores-Mir C. Mandibular asymmetry diagnosis with panoramic imaging. Am J Orthod Dentofacial Orthop. 2008 Aug; 134(2): 183-92. doi:10.1016/j.ajodo.2007.07.021 
15. Kambylafkas P, Murdock E, Gilda E, Tallents RH, Kyrkanides S. Validity of panoramic radiographs for measuring mandibular asymmetry. Angle Orthod. 2006; 76(3): 388-93. doi:

\subsection{3/0003-3219(2006)076[0388:VOPRFM]2.0.CO;2}

16. Devlin H, Yuan J. Object position and image magnification in dental panoramic radiography: a theoretical analysis. Dentomaxillofac Radiol. 2013; 42(1): 29951683. doi: $10.1259 / \mathrm{dmfr} / 29951683$

17. Larheim T, Svanaes D. Reproducibility of rotational panoramic radiography: Mandibular linear dimensions and angles. Am J Orthod Dentofacial Orthop. 1986; 90(1): 45-51.

18. Sanders D, Rigali P, Neace W, Uribe F, Nanda R. Skeletal and dental asymmetries in class II subdivision malocclusions using cone-beam computed tomography. Am J Orthod Dentofacial Orthop. 2010; 138(5): 542. e1-20. doi: 10.1016/j.ajodo.2010.02.027

19. Dawson P. Oclusión funcional: diseño de la sonrisa a partir de la ATM. Caracas Venezuela: Amolca; 2009.

20. Türp J, Vach W, Harbich K, Alt K, Strub J. Determining mandibular condyle and ramus height with the help of an orthopantomogram--a valid method J Oral Rehabil. 1996; 23(6): 395-400. 